University of Nebraska - Lincoln

DigitalCommons@University of Nebraska - Lincoln

April 2008

\title{
Imaging mechanism of piezoresponse force microscopy in capacitor structures
}

\author{
Sergei V. Kalinin \\ Materials Science and Technology Division and the Center for Nanophase Materials Science, Oak Ridge \\ National Laboratory, Tennessee, sergei2@ornl.gov \\ Brian J. Rodriguez \\ Materials Science and Technology Division and the Center for Nanophase Materials Science, Oak Ridge \\ National Laboratory, Oak Ridge, Tennessee 37831, USA, brian.rodriguez@ucd.ie \\ Seung-Hyun Kim \\ INOSTEK, Inc., Gyeonggi 426-901, Republic of Korea \\ Suk-kyoung Hong \\ Hynix Semiconductor, Inc., San 136-1, Ami-ri, Bubal-eub, Icheon-si, Kyoungki-do 467-701, Republic of \\ Korea \\ Alexei Gruverman \\ University of Nebraska-Lincoln, agruverman2@unl.edu \\ See next page for additional authors \\ Follow this and additional works at: https://digitalcommons.unl.edu/physicsgruverman \\ Part of the Physics Commons
}

Kalinin, Sergei V.; Rodriguez, Brian J.; Kim, Seung-Hyun; Hong, Suk-kyoung; Gruverman, Alexei; and Eliseev, Eugene E., "Imaging mechanism of piezoresponse force microscopy in capacitor structures" (2008). Alexei Gruverman Publications. 23.

https://digitalcommons.unl.edu/physicsgruverman/23

This Article is brought to you for free and open access by the Research Papers in Physics and Astronomy at DigitalCommons@University of Nebraska - Lincoln. It has been accepted for inclusion in Alexei Gruverman Publications by an authorized administrator of DigitalCommons@University of Nebraska - Lincoln. 
Authors

Sergei V. Kalinin, Brian J. Rodriguez, Seung-Hyun Kim, Suk-kyoung Hong, Alexei Gruverman, and Eugene E. Eliseev 


\title{
Imaging mechanism of piezoresponse force microscopy in capacitor structures
}

\author{
Sergei V. Kalinin, ${ }^{1, a)}$ Brian J. Rodriguez,, ${ }^{1, b)}$ Seung-Hyun Kim, ${ }^{2}$ Suk-Kyoung Hong, ${ }^{3}$ \\ Alexei Gruverman, ${ }^{4}$ and Eugene A. Eliseev ${ }^{5, a)}$ \\ ${ }^{1}$ Materials Science and Technology Division and the Center for Nanophase Materials Science, Oak Ridge \\ National Laboratory, Oak Ridge, Tennessee 37831, USA \\ ${ }^{2}$ INOSTEK, Inc., Gyeonggi 426-901, Republic of Korea \\ ${ }^{3}$ Hynix Semiconductor, Inc., San 136-1, Ami-ri, Bubal-eub, Icheon-si, Kyoungki-do 467-701, Republic of \\ Korea \\ ${ }^{4}$ Department of Physics and Astronomy, University of Nebraska-Lincoln, Lincoln, Nebraska 68588-0111, \\ USA \\ ${ }^{5}$ Institute for Problems of Materials Science, National Academy of Science of Ukraine, 3, Krjijanovskogo, \\ Kiev 03142, Ukraine
}

(Received 17 January 2008; accepted 13 March 2008; published online 18 April 2008)

\begin{abstract}
The image formation mechanism in piezoresponse force microscopy (PFM) of capacitor structures is analyzed. We demonstrate that the spatial resolution is a bilinear function of film and top electrode thicknesses and derive the corresponding analytical expressions. For many perovskites, the opposite contributions of $d_{31}$ and $d_{33}$ components can result in anomalous domain wall profiles. This analysis establishes the applicability limits of PFM for polarization dynamics studies in capacitors and applies them to other structural probes, including focused x-ray studies of capacitor structures. (C) 2008 American Institute of Physics. [DOI: 10.1063/1.2905266]
\end{abstract}

Applications of ferroelectric materials as nonvolatile memory components ${ }^{1,2}$ have resulted in significant interest in theoretical and experimental studies of polarization dynamics in capacitor structures. Numerous theoretical studies of polarization behavior, depolarization field and dead-layer effects, and stability of periodic domain structures by using the Ginzburg-Landau and atomistic theories are available. ${ }^{3-5}$ Experimentally, spatially resolved polarization dynamics have been addressed by focused x-ray measurements ${ }^{6}$ and piezoresponse force microscopy (PFM). ${ }^{7}$ In PFM, a periodic uniform electric field is applied between the top and bottom electrodes, and the resulting surface displacement is locally measured by a scanning probe microscope tip. ${ }^{8}$ This global excitation method is different from standard (local excitation) PFM, in which the electric field is directly applied to the tip and the local strain is detected. The PFM studies of capacitor structures have allowed the stability of uniformly switched states, ${ }^{9}$ the localization of nucleation sites ${ }^{10}$ and statistical aspects of nucleation to be studied. ${ }^{11}$ Despite the significant number of polarization imaging and switching studies in capacitors by PFM, the image formation mechanism, including parameters such as spatial resolution and information limit and the effect of the top electrode on both switching and imaging processes, has not yet been addressed. Here, we theoretically analyze the domain wall width and spatial resolution of PFM with global excitation and predict the implications for PFM-based capacitor studies.

Below, we utilize the decoupled approximation ${ }^{12,13}$ that has previously been used for the analysis of PFM with local excitation. ${ }^{14,15}$ In the geometry of the capacitor experiment

\footnotetext{
${ }^{a)}$ Authors to whom correspondence should be addressed. Electronic addresses: sergei2@ornl.gov and eliseev@i.com.ua.

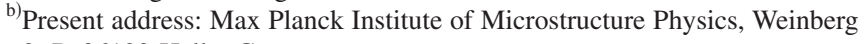
2, D-06120 Halle, Germany.
}

[Fig. 1(a)], the vertical PFM signal, i.e., the surface displacement $u_{3}(\mathbf{x})$ at location $\left(x_{1}, x_{2}, 0\right)$ is

$$
\begin{aligned}
u_{3}\left(x_{1}, x_{2}, 0\right)= & \int_{-\infty}^{\infty} d \xi_{1} \int_{-\infty}^{\infty} d \xi_{2} \int_{L}^{L+H} d \xi_{3} \frac{\partial G_{3 j}\left(-\xi_{1},-\xi_{2}, \xi_{3}\right)}{\partial \xi_{k}} \\
& \times c_{k j m n} E_{3} d_{3 n m}\left(x_{1}+\xi_{1}, x_{2}+\xi_{2}, \xi_{3}\right) .
\end{aligned}
$$

The coordinates $\mathbf{x}=\left(x_{1}, x_{2}, x_{3}\right)$ are linked to the sample [Fig. 1(a)]. Coefficients $d_{m n k}$ are position dependent components of the piezoelectric strain tensor, $c_{j l m n}$ are elastic stiffness constants tensors, and $E_{k}$ is the external electric field components. Green's function for a semi-infinite medium $G_{3 j}(\mathbf{x}, \boldsymbol{\xi})$ links the eigenstrains $c_{j l m n} d_{m n k} E_{k}$ to the displacement field. Here, we use an approximation of elastically isotropic media for Green's function. ${ }^{16,17}$

By considering the domain structure with two opposite domains divided by a flat wall perpendicular to the film surface, ${ }^{18}$ we suppose the following distribution:

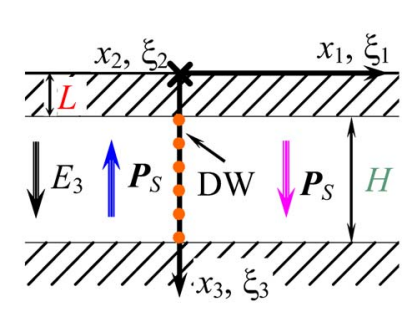

(a)

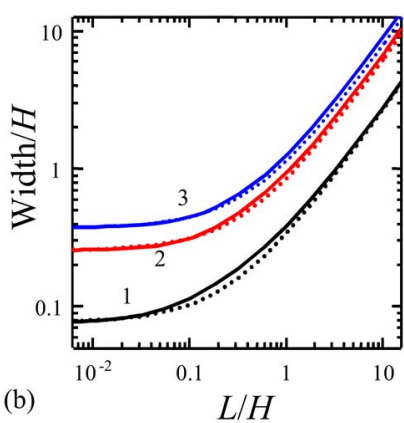

(b)

FIG. 1. (Color online) Coordinate systems in global excitation PFM experiment. (b) Domain wall width on level $\eta=0.76$ as a function of the top electrode thickness for $\nu=0.3$ and $-d_{33} / d_{31}=2,3$, and 4 (curves 1,2 , and 3 ). Solid curves represent the numerical calculations; dashed curves are after the approximations $(5 \mathrm{a})-(5 \mathrm{c})$. 

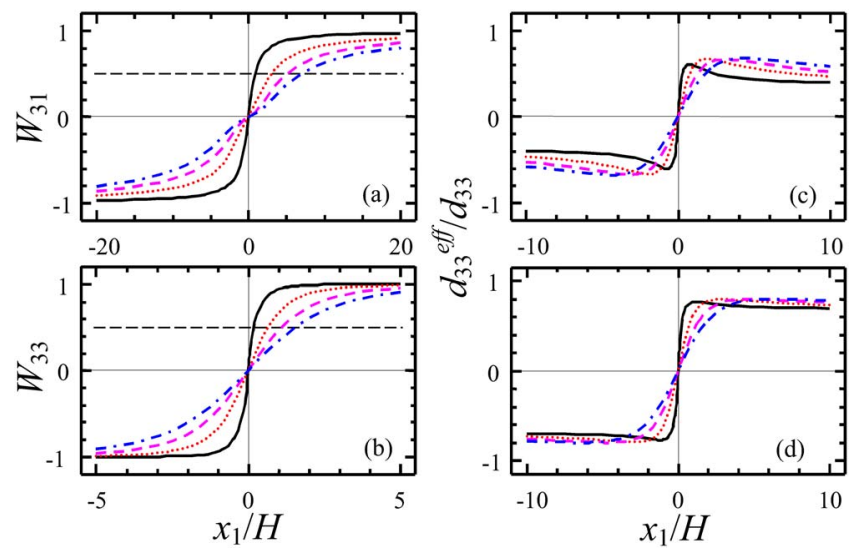

FIG. 2. (Color online) Coordinate dependence of (a) $d_{31}$ and (b) $d_{33}$ contribution to the vertical PFM response for $\nu=0.3$ and different thicknesses of top electrode $L / H=0,1,2$, and 3 (solid, dotted, dashed, and dash-dotted curves). Profiles of the vertical PFM response across the domain wall for $\nu=0.3,-d_{33} / d_{31}=2.5,5[(\mathrm{c})$ and (d) $]$, and different thicknesses of top electrode $L / H=0,1,2$, and 3 (solid, dotted, dashed, and dash-dotted curves).

$$
d_{i n m}\left(\xi_{1}, \xi_{2}, \xi_{3}\right)= \begin{cases}0, & \xi_{3}>L+H \cup \xi_{3}<L \\ -d_{i \alpha}, & L<\xi_{3}<L+H \cap \xi_{1}<0 \\ d_{i \alpha}, & L<\xi_{3}<L+H \cap \xi_{1}>0 .\end{cases}
$$

Here, $L$ and $H$ are the thickness values of the top electrode and film, respectively. Vertical displacement [Eq. (1)] can be rewritten as follows:

$$
\begin{aligned}
u_{3}\left(x_{1}, x_{2}, 0\right)= & -U\left[W_{33}\left(x_{1}, L, H\right) d_{33}+(1\right. \\
& \left.+2 \nu) W_{31}\left(x_{1}, L, H, \nu\right) d_{31}\right] .
\end{aligned}
$$

Here, $U=H E_{3}$ is the potential difference between the top and the bottom electrodes, and $\nu$ is the Poisson ratio. After the cumbersome integration of Eq. (1) with distribution (2), functions $W_{33}$ and $W_{31}$ are analytically derived as ${ }^{19}$

$$
\begin{aligned}
& W_{33}\left(x_{1}, L, H\right)=f_{1}\left(x_{1}, L, H\right)+f_{2}\left(x_{1}, L, H\right), \\
& W_{31}\left(x_{1}, L, H, \nu\right)=f_{1}\left(x_{1}, L, H\right)+\frac{\nu}{2(1+2 \nu)} f_{2}\left(x_{1}, L, H\right),
\end{aligned}
$$

where the following designations are introduced:

$$
\begin{aligned}
f_{1}\left(x_{1}, L, H\right)= & \frac{2}{\pi}\left[\left(\frac{L}{H}+1\right) \arctan \left(\frac{x_{1}}{L+H}\right)\right. \\
& \left.-\left(\frac{L}{H}\right) \arctan \left(\frac{x_{1}}{L}\right)\right], \\
f_{2}\left(x_{1}, L, H\right)= & \frac{2}{\pi} \frac{x_{1}}{H} \ln \left[\frac{(L+H)^{2}+x_{1}^{2}}{L^{2}+x_{1}^{2}}\right] .
\end{aligned}
$$

Far from the wall, $x_{1} \rightarrow \pm \infty$, functions [Eqs. (4a) and (4b)] tend to $W_{3 i}\left(x_{1}, L, H\right) \rightarrow \pm 1$. Hence, the piezoresponse signal in a uniformly poled capacitor is $u_{3}\left(x_{1}, x_{2}, 0\right) / U=$ $-d_{33}-(1+2 \nu) d_{31}$. The nonzero contribution of $d_{31}$ is directly related to the mechanical conditions on the boundary between the film and the bottom electrode, which are assumed to have the same mechanical properties. The dependence of functions $W_{3 i}$ on coordinate $x_{1}$ is shown in Figs. 2(a) and 2(b). Note that the component profile width is much higher
TABLE I. Width derivatives on $H$ and $L, w=a H+b L$, for $\eta=0.76, \nu=0.3$.

\begin{tabular}{ccccc}
\hline \hline Material & $d_{33}(\mathrm{pm} / \mathrm{V})$ & $d_{31}(\mathrm{pm} / \mathrm{V})$ & $a$ & $b$ \\
\hline $\mathrm{BaTiO}_{3}$ & 86 & -35 & 0.17 & 0.45 \\
$\mathrm{PbTiO}_{3}$ & 117 & -25 & 0.43 & 0.81 \\
$\mathrm{PZT6B}_{(111)} \mathrm{PZT40/60}^{\mathrm{a}}$ & 75 & -29 & 0.19 & 0.49 \\
$\mathrm{LiNbO}_{3}$ & 73.5 & -24 & 0.26 & 0.60 \\
$\mathrm{LiTaO}_{3}$ & 6 & -1 & 0.51 & 0.90 \\
\hline
\end{tabular}

${ }^{\mathrm{a}}$ Reference 21 .

for $W_{31}$ than for $W_{33}$, which is related to the smaller contribution of $f_{2}$ to $W_{31}$. As the consequence of the different widths of $W_{31}$ and $W_{33}$, the full PFM response profile [Eq. (3)] exhibits an anomalous shape with a minimum and a maximum near the wall center for the most encountered case of $d_{31} / d_{33} \approx-0.5--0.3$ [see Figs. 2(c) and 2(d)]. At the same time, the slope of the response at the wall center is primarily determined by $d_{33}$. As the $d_{31}$ absolute value increases, the saturation level decreases much faster than the slope, so that the width also decreases.

Here, we define the generalized domain wall width, or PFM resolution, as the distance between the points at which the response is saturated to the fraction $\eta$ of the signal at infinity. The width can be approximated as a bilinear function, $w(L, H) \cong a H+b L$, of the film and top electrode thicknesses,

$$
w(L, H) \cong a\left(\eta, \nu, \frac{d_{31}}{d_{33}}\right) H+b\left(\eta, \nu, \frac{d_{31}}{d_{33}}\right) L,
$$

where coefficient $a$ is the solution of the transcendental equation,

$$
\arctan \left(\frac{a}{2}\right)+\frac{d_{33}+\nu d_{31} / 2}{d_{33}+(1+2 \nu) d_{31}} \ln \left(1+\frac{4}{a^{2}}\right) \frac{a}{2}=\frac{\pi}{2} \eta
$$

and coefficient $b$ is

$$
b=\eta \frac{\pi}{2} \frac{d_{33}+(1+2 \nu) d_{31}}{d_{33}+\nu d_{31} / 2} .
$$

Typical values for coefficient $a$ varies from 0.08 to 0.4 at $\eta=0.76$ and $-d_{33} / d_{31}=2-4$ (see Table I). The dependence of width on top electrode thickness obtained by the numerical calculation and with the help of Eq. (5a)-(5c) is shown in Fig. 1(b). The immediate consequence of Eq. (5a) is that for a mechanically uniform capacitor (no cracks, etc.), the signal generation area in PFM, even for point mechanical contact, is determined by the total thickness of the capacitor structure. Note that the domain wall width linearly scales with sample thickness for thin top electrodes $(W / H=0.08-0.3$, depending on piezoelectric anisotropy) and rapidly increases with top electrode thickness. This agrees with experimental data obtained at several capacitor thicknesses summarized in Table II for (111)-oriented $180 \mathrm{~nm}$ thick film of lead zirconate titanate $\mathrm{Pb}(\mathrm{Zr}, \mathrm{Ti}) \mathrm{O}_{3} \quad(\mathrm{PZT}) \quad 40 / 60$ and reported elsewhere. ${ }^{20}$ Note that this conclusion can readily be understood as a consequence of the presence of the primary length scale in the system, and similar behavior is anticipated for, e.g., synchrotron x-ray-based measurements of domain structures.

For cases when the domain wall is also associated with the grain boundary and the grains are mechanically decou- 
TABLE II. Experimental and theoretical domain wall widths for different top electrode thicknesses.

\begin{tabular}{cccc}
\hline \hline Top electrode thickness & Poled cap & Unpoled cap & Theor. \\
\hline 50 & $130-170$ & $40-60$ & 81 \\
150 & & 80 & 150 \\
250 & $180-250$ & & 217 \\
\hline \hline
\end{tabular}

pled, the coefficient in Eq. (5a)-(5c) is $a=0$. Here, the top electrode thickness is the only relevant length scale. In the case of intermediate coupling, the bulk contribution will be reduced, but the top electrode contribution is expected to be constant. Finally, for the liquid top electrode (imaging in conductive solution), $b=0$.

To summarize, we have analyzed the imaging mechanism in PFM with global excitation. The analytical expressions for the domain wall profile are derived. The resolution, i.e., the measured domain wall profile is derived for an infinitely thin wall and a point mechanical contact approximation. It is shown that domain wall width linearly scales with the thickness of the ferroelectric layer and top electrode, although with significantly different slopes. For typical material parameters and thin top electrode $(L \ll H)$, the resolution is expected to be $w \sim 0.2 \cdot H$, which presents the ultimate limit on PFM resolution in capacitors imposed by the presence of the characteristic length scale. Similar behavior is anticipated for other probes of the domain wall, including focused $x$ ray. Higher resolutions can be only observed if walls are mechanically uncoupled or as a result of the cross talk between topography and PFM signal, unrelated to domain structures.

The research was sponsored in part (S.V.K. and B.J.R.) by the Center for Nanophase Materials Sciences, U.S. Department of Energy under Contract No. DE-AC0500OR22725 with Oak Ridge National Laboratory, managed and operated by UT-Battelle, LLC. One of the authors
(B.J.R.) acknowledges the support of the Alexander von Humboldt Foundation.

${ }^{1}$ J. Scott, Ferroelectric Memories (Springer, Berlin, 2000).

${ }^{2}$ Nanoelectronics and Information Technology, edited by R. Waser (WileyVCH, New York, 2003).

${ }^{3}$ N. A. Pertsev and H. Kohlstedt, Phys. Rev. Lett. 98, 257603 (2007).

${ }^{4}$ M. Stengel and N. A. Spaldin, Nature (London) 443, 679 (2006).

${ }^{5}$ A. M. Bratkovsky and A. P. Levanyuk, Phys. Rev. Lett. 94, 107601 (2005).

${ }^{6}$ A. Gregoriev, D.-H. Do, D. M. Kim, C.-B. Eom, B. Adams, E. M. Dufresne, and P. G. Evans, Phys. Rev. Lett. 96, 187601 (2006).

${ }^{7}$ A. Gruverman and A. Kholkin, Rep. Prog. Phys. 69, 2443 (2006).

${ }^{8}$ J. A. Christman, R. R. Woolcott, A. I. Kingon, and R. J. Nemanich, Appl. Phys. Lett. 73, 3851 (1998).

${ }^{9}$ A. Gruverman, B. J. Rodriguez, A. I. Kingon, R. J. Nemanich, J. S. Cross, M. Tsukada, and Y. Horii, Appl. Phys. Lett. 82, 3071 (2003).

${ }^{10}$ A. Gruverman, B. J. Rodriguez, C. Dehoff, A. I. Kingon, and R. J. Nemanich, Appl. Phys. Lett. 87, 082902 (2005).

${ }^{11}$ D. J. Kim, J. Y. Jo, T. H. Kim, S. M. Yang, B. Chen, Y. S. Kim, and T. W. Noh, Appl. Phys. Lett. 91, 132903 (2007).

${ }^{12}$ F. Felten, G. A. Schneider, J. M. Saldaña, and S. V. Kalinin, J. Appl. Phys. 96, 563 (2004).

${ }^{13}$ D. A. Scrymgeour and V. Gopalan, Phys. Rev. B 72, 024103 (2005).

${ }^{14}$ E. A. Eliseev, S. V. Kalinin, S. Jesse, S. L. Bravina, and A. N. Morozovska, J. Appl. Phys. 102, 014109 (2007).

${ }^{15}$ A. N. Morozovska, E. A. Eliseev, S. L. Bravina, and S. V. Kalinin, Phys. Rev. B 75, 174109 (2007).

${ }^{16}$ A. I. Lur'e, Three-Dimensional Problems of the Theory of Elasticity (Interscience, New York, 1964).

${ }^{17}$ L. D. Landau and E. M. Lifshitz, Theory of Elasticity, Theoretical Physics Vol. 7 (Butterworth-Heinemann, Oxford, 1976).

${ }^{18}$ Note that in polycrystalline or textured capacitors, only the "effective domains" could be defined as the ensemble of grains with similar net polarizations out of plane.

${ }^{19}$ S. V. Kalinin, B. J. Rodriguez, S.-H. Kim, S.-K. Hong, A. Gruverman, and E. A. Eliseev, e-print, arXiv:0801.2568.

${ }^{20}$ T. Jungk, A. Hoffmann, and E. Soergel, J. Appl. Phys. 102, 084102 (2007).

${ }^{21}$ Piezoelectric modules were recalculated from values for (100) oriented PZT40/60 given by M. J. Haun, E. Furman, S. J. Jang, and L. E. Cross, Ferroelectrics 99, 63 (1989). 